\section{Case Reports in Oncology}

\title{
Central Nervous System Peripheral T Cell Lymphoma Manifesting as Lymphomatosis Cerebri That Was Misdiagnosed as Neuro-Behçet's Disease: A Case Report
}

\author{
Osamu Imataki Shumpei Uchida Shigeyuki Yokokura Makiko Uemura \\ Norimitsu Kadowaki
}

Division of Hematology, Department of Internal Medicine, Faculty of Medicine, Kagawa University, Kagawa, Japan

\section{Keywords}

Malignant lymphoma $\cdot$ Behçet's disease $\cdot$ Peripheral T cell lymphoma $\cdot$ Not otherwise specified · Lymphomatosis cerebri · Primary central nerves lymphoma

\begin{abstract}
Background: Lymphomatosis cerebri (LC) is a unique form of primary central nerves lymphoma (PCNSL), which presents as diffuse infiltration of lymphoma cells characteristically in the white matter rather than tumor formation. However, the involvement of central nervous system (CNS) is unclear because of the lack of contrast enhancement. Case Presentation: We treated a 53-year-old woman with LC and brain lesions resembling neuro-Behcet's disease. She had a past history of acute uveitis and current symptoms of somnolence and gait disturbances progressing for one month. Cranial magnetic resonance imaging (MRI) revealed high signal lesions in the brain stem. Based on her past history and present clinical findings, she was clinically diagnosed with neuro-Behcet's disease, which was treated with $1 \mathrm{~g}$ of methylprednisolone (mPSL) pulse therapy. Repeated mPSL pulse therapy resulted in a minor response, but the cerebral lesions persisted. After a short remission of several months, signal changes of the brain stem lesion recurred and her consciousness level worsened at 4 months after diagnosis.
\end{abstract}


Upon admission to our hospital, positron emission tomography with 2-deoxy-2-[fluorine18]fluoro-D-glucose integrated with computed tomography revealed abnormal uptake in the systemic lymph nodes (LNs), including the bilateral inguinal LNs. A diagnosis based on a biopsy of the left inguinal LNs was primary central nervous system lymphoma with inguinal LN lesions, manifesting as LC from malignant peripheral T cell lymphoma, not otherwise specified. Four courses of high-dose methotrexate $\left(3.5 \mathrm{~g} / \mathrm{m}^{2}\right)$ therapy lead to temporary recovery of consciousness, but there was no improvement in other neurological findings. All nodal lesions tentatively regressed. Serum soluble interleukin-2 receptor (sIL-2R) (normal range: 121-613 $\mathrm{U} / \mathrm{mL}$ ) was constitutively decreased from $8,520 \mathrm{U} / \mathrm{mL}$ before chemotherapy to $740 \mathrm{U} / \mathrm{mL}$ after chemotherapy. We observed cerebral micro-bleeds in the center of LC lesions during chemotherapy, but no surgical intervention was required. Two months later, LC recurred in the brain, which was fatal. Conclusions: Neuro-Behçet's disease is difficult to distinguish from LC when other clinical findings, including human leukocyte antigen disparity, serum sIL-2R, or cerebrospinal IL-6, are lacking. LC should be differentiated from CNS lymphoma before corticosteroid therapy.

(C) 2018 The Author(s)

Published by S. Karger AG, Basel

\section{Background}

Primary central nervous system lymphoma (PCNSL) is an extranodal high-grade lymphoma with mature B cell neoplasms accounting for $90 \%$ of cases and mature T cell lymphoma-derived PCNSL accounting for 10\% [1]. Lymphomatosis cerebri (LC) is a rare variant of PCNSL characterized by a diffusely infiltrating pattern without contrast enhancement [2], regardless of the cell origin of lymphocytes (B or T cell neoplasm). LC shows pathologically perivascular lymphocyte infiltration by both lymphoma cells and normal reactive lymphocytes, which pathologically resembles encephalitis [3]. The clinical symptoms and manifestations of PCNSL correspond to this pathology. We encountered a LC patient who was initially treated for neuro-Behçet's disease, but failed to achieve disease remission. We should notify LC lesions in the brain are similar to the neuro-Behçet's disease.

\section{Case Presentation}

A 53-year-old woman had initially consulted a neurologist for treatment of somnolence and gait disturbances that had progressed for a period of one month. Cranial magnetic resonance imaging (MRI) revealed a high signal lesion in the brain stem. Her past history of uveitis and concurrent anterior chamber uveitis lead to a diagnosis of neuro-Behçet's disease. She received corticosteroid pulse therapy with $1 \mathrm{~g}$ of methylprednisolone (mPSL) per day for consecutive 3 days. Since the first mPSL pulse therapy resulted in a minor improvement of the neurological symptoms, two additional courses of mPSL pulse therapy were administered, which resulted in only a partial response. In addition, the cerebral lesions had remained and the signal changes of the brain stem lesion had progressed afterward. Since her consciousness level worsened at 4 months after diagnosis, she was admitted to our hospital.

On admission, her neurological Glasgow coma score was E3 V3 M5. The light reaction of the bilateral eyes was attenuated and dysarthria was observed. The limb manual muscle test was grade 3 to 4 with increased tendon reflex and positive Babinski signs bilaterally. At that time, a number of swollen lymph nodes were palpable in the right inguinal region. Laboratory testing showed that serum soluble interleukin-2 receptor (sIL-2R) levels were highly elevated 
to 5,512 U/mL (normal range, 135-483 U/mL). Cranial MRI showed diffuse T2 high signal areas (Fig. 1) in the cerebellum, brainstem, and peri-lateral ventricle. However, contrast-enhanced MRI detected no contrast lesion. Positron emission tomography with 2-deoxy-2-[fluorine-18]fluoro-D-glucose integrated with computed tomography (18F-FDG-PET) revealed abnormal accumulation in the left cervix, mediastinum, pelvis, and bilateral inguinal lymph nodes (Fig. 2). A biopsy of the lymph nodes in the left inguinal region suggested a diagnosis of malignant lymphoma or peripheral T cell lymphoma, not otherwise specified (Fig. 3). There was no evidence of lymphoma cell infiltration of the intramedullary and cerebrospinal fluid. Ophthalmological findings confirmed the presence of acute uveitis in the anterior chamber. Right post-corneal kelatic precipitation and anterior chamber flares were also observed. Rubeosis iridis was not observed, thus diabetic retinopathy and age-related macular degeneration were excluded.

We diagnosed her PCNSL manifesting as LC with inguinal LN lesions. Histological diagnosis was peripheral $\mathrm{T}$ cell lymphoma, not otherwise specified, based on a biopsy of the left inguinal LNs. Although PCNSL was diagnosed, a brain biopsy was not performed. Four courses of high-dose methotrexate $\left(3.5 \mathrm{~g} / \mathrm{m}^{2}\right)$ therapy were administered, which resulted in temporary recovery of consciousness, but no improvement to the other neurological findings. Although cerebral micro-bleeds were repeatedly observed in the center of LC lesions during chemotherapy, no neurosurgical intervention was required. All nodal lesions were ancillary in tentative regression. The brain lesion was difficult to evaluate by MRI because of remaining degenerative signals and no clear lesions were found. Serum IL-2R (normal range: 121-613 $\mathrm{U} / \mathrm{mL}$ ) was constitutively decreased from $8,520 \mathrm{U} / \mathrm{mL}$ before chemotherapy to $740 \mathrm{U} / \mathrm{mL}$ after chemotherapy. Hence, the initial treatment was terminated. Two months later, the consciousness disturbances and progressed and cranial MRI detected recurrent lesions in the brain. The patient refused human leukocyte antigen evaluation and eventually died from LC.

\section{Discussion}

We encountered a patient with brain lesions due to central nervous lymphoma manifesting as LC. After four courses of high-dose methotrexate therapy, the lymph node lesions had improved, but the brain lesion remained. She had a past history of acute uveitis, which resulted in a misdiagnosis of malignant disease. Neuro-Behçet's disease affects the CNS with consciousness disturbances, dementia, pyramidal and extra-pyramidal signs, and hemiparesis [4]. However, the neurological complications of Behçet's disease have not been clearly characterized [5]. Parenchymal brain disease is more common and mainly affects the brain stem and basal ganglia. On MRI, lesions of the white matter region, brain stem, and basal ganglia are frequently visible. Since such imaging findings are specific to neuro-Behçet's disease, it is rather difficult to distinguish LC-associated lesions [6]. There has only been one report of malignant lymphoma masquerading as cutaneous and enteral lesions of Behçet's disease in the literature, which described a case of malignant lymphoma mimicking Behçet's disease because of the similarities between these diseases [7]. The present case had resembled Behçet's disease-like mucocutaneous lesions. To the best of our knowledge, this is the first report of malignant lymphoma masquerading as neuro-Behçet's disease.

A shortcoming of this case report is the lack of pathological evidence of CNS lesions. Nonetheless, an incidental complication of malignant lymphoma and Behçet's disease should be considered in such cases. In regard to this possibility, only a single case of malignant lymphoma complicated with Behçet's disease has been reported in the English literature to date 
[8]. This report referenced 18 cases of Behçet's disease concomitant to malignancy, including lymphoma, but did not discuss a direct association between these diseases. In terms of collagen diseases, an association between carcinogenesis and immunosuppressant use is a possible mechanism. Indeed, Behçet's disease treated with chlorambucil increases the risk of malignancy [9]. A literature search of only abstracts in the PubMed database retrieved several case reports [10-12] suggesting that malignant lymphoma and Behçet's disease should be diagnosed independently by pathological examination. There are no etiological or pathogenic associations between these two diseases, thus the underlying mechanism in the present case remains unclear. It is also unclear whether uveitis was a specific finding to Behçet's disease or lymphoma, although it is less likely that uveitis was caused by infiltration of lymphoma cells. For cases of LC with white matter lesions, as with the present case, it would be helpful to exclude neuro-Behçet's disease by analysis of sIL-2R in the serum and IL-6 in the cerebrospinal fluid [13].

Taken together, the possibility of Behçet's disease in this case is quite low because the treatment course did not indicate the presence of Behçet's disease. Serological testing for malignant lymphoma was synchronized to disease progression. The rapid progression and poor prognosis of this case suggested malignancy. The presence of uveitis is unspecific when diagnosing Behçet's disease. LC from CNS lymphoma should be differentiated before administration of corticosteroid therapy.

\section{Statement of Ethics}

Ethics approval and consent to participate: Institutional review board (IRB) approved the case report and submission of medical literature. We obtained the approval from the IRB.

Consent to publish: An informed consent was obtained from the patient for the publication of their information and imaging.

\section{Availability of Data and Materials}

All clinical data and imaging to diagnose and discuss this case are included in this published article.

\section{Disclosure Statement}

The authors declare that they have no conflicts of interest.

\section{Funding Sources}

We don't have any funding source to disclose concerning to this report. 


\section{Author Contributions}

OI and SU managed the patient's case and drafted the manuscript. SY and MU contributed to the literature search and interpreted the result. MU managed the data and suggested important intellectual content. OI and SU reviewed the manuscript. MU made substantial contributions to the concept and design of this report. NK organized and managed this work. All authors read and approved the final version of the manuscript.

\section{References}

1 Grommes C, DeAngelis LM. Primary CNS Lymphoma. J Clin Oncol. 2017 Jul;35(21):2410-8.

2 Bakshi R, Mazziotta JC, Mischel PS, Jahan R, Seligson DB, Vinters HV. Lymphomatosis cerebri presenting as a rapidly progressive dementia: clinical, neuroimaging and pathologic findings. Dement Geriatr Cogn Disord. 1999 Mar-Apr;10(2):152-7.

3 Rollins KE, Kleinschmidt-DeMasters BK, Corboy JR, Damek DM, Filley CM. Lymphomatosis cerebri as a cause of white matter dementia. Hum Pathol. 2005 Mar;36(3):282-90.

4 Mendes D, Correia M, Barbedo M, Vaio T, Mota M, Gonçalves O, et al. Behçet's disease-a contemporary review. J Autoimmun. 2009 May-Jun;32(3-4):178-88.

5 Kidd D, Steuer A, Denman AM, Rudge P. Neurological complications in Behçet's syndrome. Brain. 1999 Nov;122(Pt 11):2183-94.

6 Yu H, Gao B, Liu J, Yu YC, Shiroishi MS, Huang MM, et al. Lymphomatosis cerebri: a rare variant of primary central nervous system lymphoma and MR imaging features. Cancer Imaging. 2017 Oct;17(1):26.

7 Lee H, Kim SH, Lee SW, Zheng Z, Bang D, Kim DY. A case of extranodal natural killer/T-cell lymphoma mimicking refractory Behçet's disease. Acta Derm Venereol. 2015 Apr;95(4):491-2.

8 Abe T, Yachi A, Yabana T, Ishii Y, Tosaka M, Yoshida Y, et al. Gastric non-Hodgkin's lymphoma associated with Behçet's disease. Intern Med. 1993 Aug;32(8):663-7.

9 Hamza MH. Cancer complicating Behçet's disease treated with chlorambucil. Ann Rheum Dis. 1986 Sep;45(9):789.

10 Huang Q, Lai W, Yang M. [Behcet's disease complicated by malignant lymphoma: a case report and literature review]. Nan Fang Yi Ke Da Xue Xue Bao. 2013 Jun;33(6):934-6.

11 Houman MH, Ben Ghorbel I. B'Chir-Hamzaoui S, Lamloum M, Kchir N, Miled M [Intestinal lymphoma associated with Behçet disease]. Ann Med Interne (Paris). 2001;152:415-8.

12 Harada K, Ohtsuru K, Nakayama K, Takagi S, Sugita Y, Torigoe R. [Intracranial primary malignant lymphoma following Behçet's disease-case report]. No To Shinkei. 1992 Nov;44(11):1029-33.

13 Akman-Demir G, Tüzün E, Içöz S, Yeşilot N, Yentür SP, Kürtüncü M, et al. Interleukin-6 in neuro-Behçet's disease: association with disease subsets and long-term outcome. Cytokine. 2008 Dec;44(3):373-6. 


\section{Case Reports in Oncology}

Imataki et al: Central Nervous System Peripheral T Cell Lymphoma Manifesting as Lymphomatosis Cerebri That Was Misdiagnosed as Neuro-Behçet's Disease

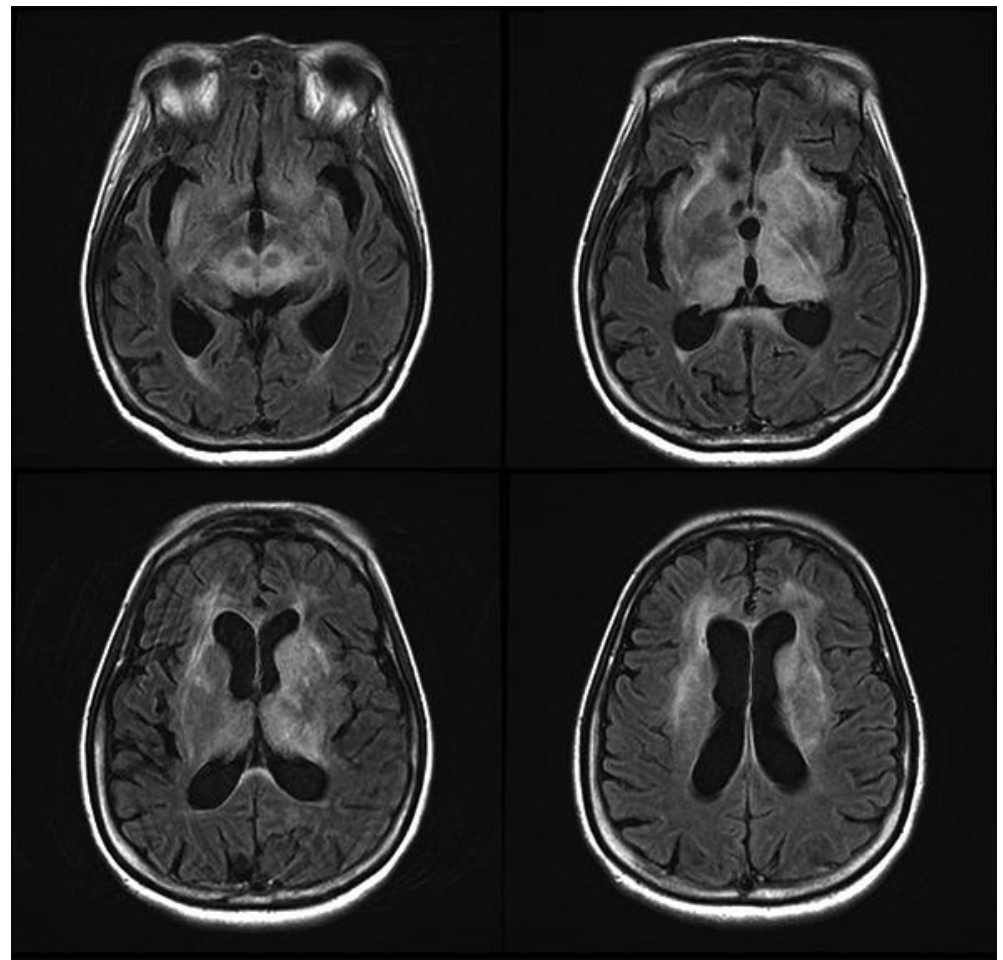

Fig. 1. Cranial MRI showing diffuse T2 high signal areas in the cerebellum, brainstem, and peri-lateral ventricle, while contrast-enhanced MRI detected no contrast lesion. 


\section{Case Reports in Oncology}

Imataki et al: Central Nervous System Peripheral T Cell Lymphoma Manifesting as Lymphomatosis Cerebri That Was Misdiagnosed as Neuro-Behçet's Disease
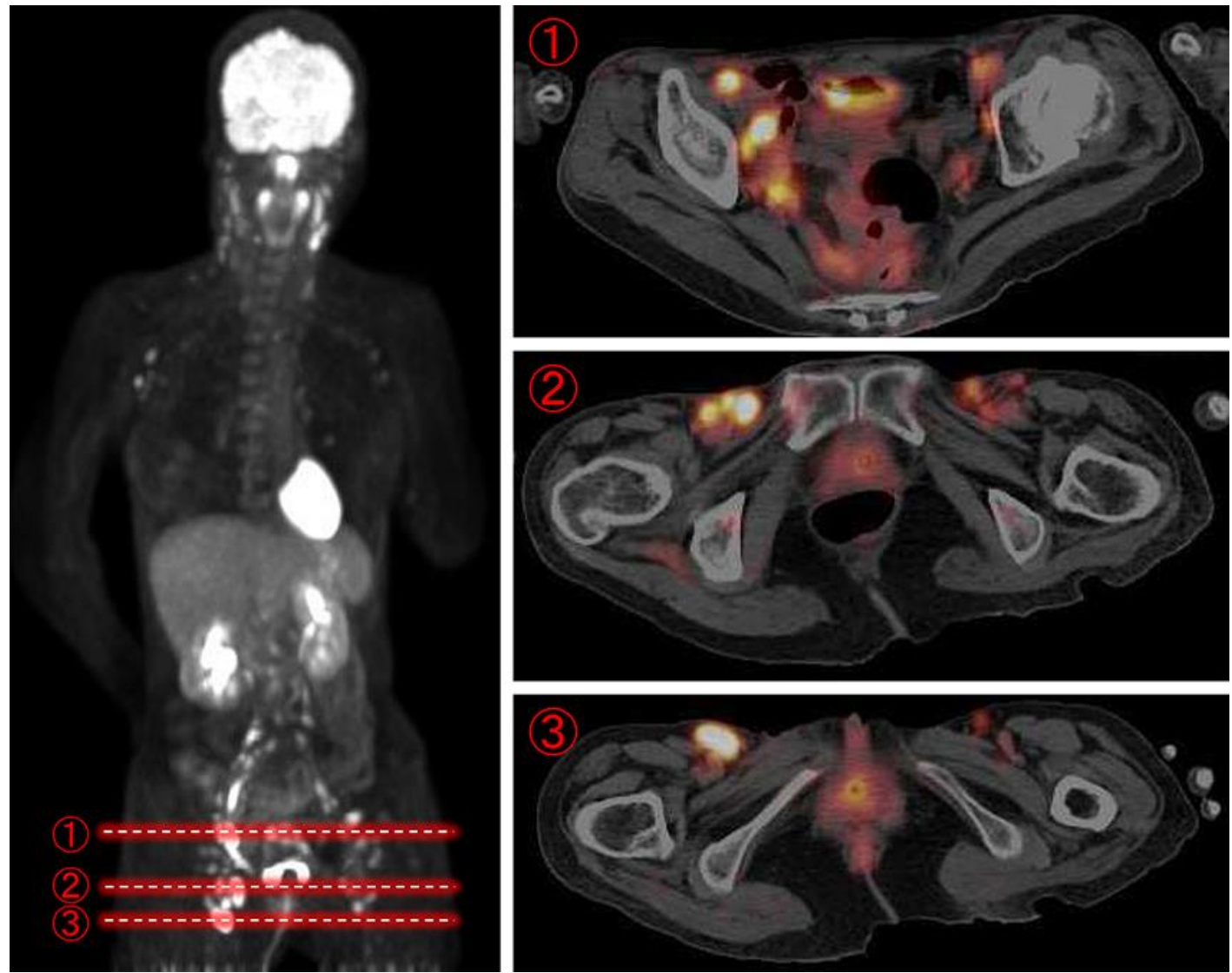

Fig. 2. 18F-FDG-PET revealed abnormal uptake in the left cervix, mediastinum, pelvis, and bilateral inguinal lymph nodes. 


\section{Case Reports in Oncology}

\begin{tabular}{l|l}
\hline Case Rep Oncol 2018;11:806-813 \\
\hline DOI: 10.1159/000495033 & $\begin{array}{l}\text { c 2018 The Author(s). Published by S. Karger AG, Basel } \\
\text { www.karger.com/cro }\end{array}$ \\
\hline
\end{tabular}

Imataki et al: Central Nervous System Peripheral T Cell Lymphoma Manifesting as Lymphomatosis Cerebri That Was Misdiagnosed as Neuro-Behçet's Disease
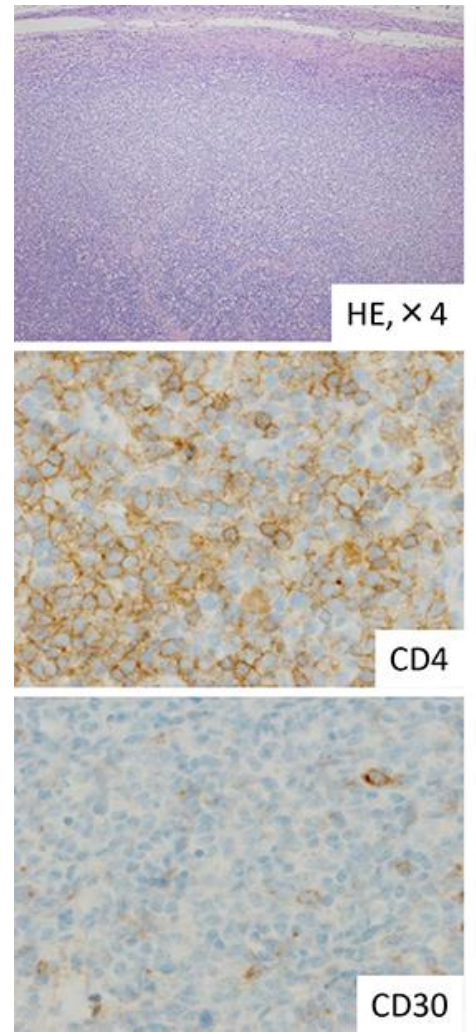
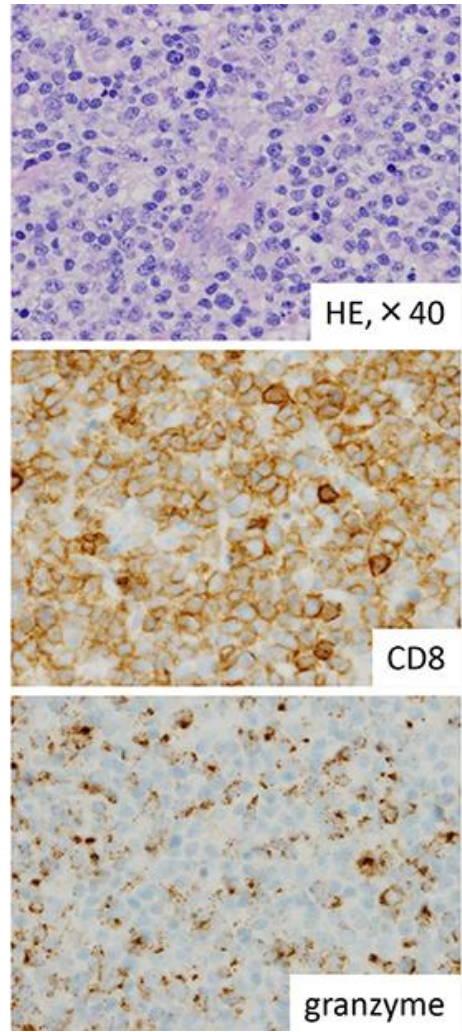
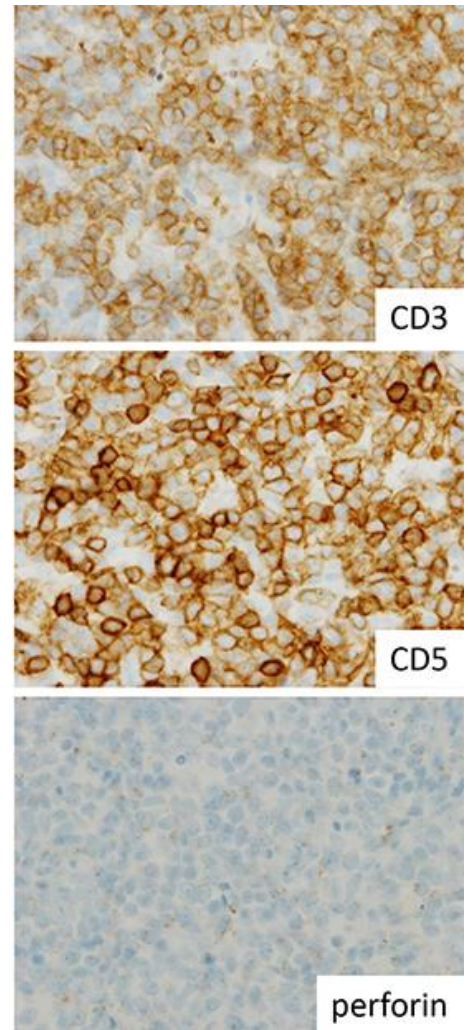

Fig. 3. Lymph node biopsy in the left inguinal region revealed malignant lymphoma, pathologically diagnosed as peripheral T cell lymphoma, not otherwise specified. Immunohistochemical analysis found that the tumor cells were positive for $\mathrm{CD} 3, \mathrm{CD} 4, \mathrm{CD} 8, \mathrm{CD} 5$, weekly $\mathrm{CD} 30$, and granzyme $\mathrm{B}$, and partially positive for perforin. 